\title{
Variación morfológica y dieta en dos poblaciones de Liolaemus elongatus Koslowsky, 1896 (Iguania: Tropiduridae) del noroeste patagónico
}

\author{
Morphological variation and diet in two Liolaemus elongatus Koslowsky, 1896 \\ (Iguania: Tropiduridae) populations from northwestern Patagonia
}

\author{
RAQUEL QUATRINI ${ }^{1,2}$, ADRIANA ALBINO³ $\&$ MÓNICA BARG
}

\author{
${ }^{1}$ Centro Regional Universitario Bariloche, Unidad Postal Universidad del Comahue, \\ 8400 San Carlos de Bariloche, Argentina \\ ${ }^{2}$ Dirección actual: Facultad de Ciencias, Universidad de Chile, Las Palmeras 3425, Santiago, \\ Chile,e-mail: rquatrin@codon.ciencias.uchile.cl \\ ${ }^{3}$ Consejo Nacional de Investigaciones Científicas y Tecnológicas y Departamento de Biología, \\ Universidad Nacional de Mar del Plata, Funes 3250, 7600 Mar del Plata, Argentina, \\ e-mail: aalbino@mdp.edu.ar
}

\begin{abstract}
RESUMEN
Se estudió morfología y dieta de dos poblaciones de Liolaemus elongatus del noroeste de la Patagonia, una de ambiente de estepa y otra de bosque. Ambas poblaciones se encuentran separadas por una distanciada de $30 \mathrm{~km}$. Se evaluó la variación entre poblaciones y entre sexos de la coloración y patrón de diseño corporal, los parámetros métricos más relevantes (longitud hocico-cloaca, longitud y ancho de la cabeza y longitud del maxilar), así como de la composición y diversidad de los contenidos digestivos de 22 ejemplares adultos de estepa y 25 de bosque. Las poblaciones difirieron en la coloración, el patrón de diseño y en el tamaño corporal promedio (mayor en la zona de estepa). La variación en la coloración podría estar relacionada con factores genéticos o selectivos. Los valores mayores de tamaño corporal en la estepa podrían representar una disminución de tamaño a mayor altitud. No se encontró dimorfismo sexual en la coloración ni en la longitud hocico-cloaca en ninguna de las dos poblaciones. Respecto a la dieta, se advirtió un predominio de consumo de formícidos en ambas poblaciones. Los vegetales resultaron ser un componente usual, contrastando con las opiniones previas que consideraban a la especie exclusivamente insectívora. La disponibilidad de elementos vegetales podría ser un factor de considerable importancia para determinar la tendencia de esta especie a la omnivoría. Las diferencias en la dieta entre las dos poblaciones analizadas podrían estar relacionadas con diferentes parámetros ecológicos y/o con características intrínsecas de las poblaciones.
\end{abstract}

Palabras clave: dieta, Liolaemus elongatus, morfología, Tropiduridae.

\begin{abstract}
Morphology and diet of two Liolaemus elongatus populations from northwestern Patagonia, consisting of a steppe and a forested patch, and $30 \mathrm{~km}$ apart from each other, were studied. The variation between populations and sexes of the coloration and design pattern, the most relevant metrical parameters, and that of composition and diversity of the digestive contents was examined in 22 steppe and 25 forest adult specimens. Populations differed in coloration, design pattern and average body size, with individuals from steppe being larger. Variation in color could be related to either genetic or selective factors. The lower altitude of the steppe zone might account for the bigger size of individuals therein. No sexual dimorphism was detected either in coloration or body size in individuals of both populations. Regarding diet, individuals from both populations appeared to feed mainly on ants (Formicidae). Nonetheless, plant material was frequent, which contradicts previous opinions that considered the species as being exclusively insectivorous. Availability of plants could therefore be a major influence on the tendency of this species towards omnivory. Differences in diet between the two populations could be related to different ecological parameters and/or to intrinsic characteristics of the populations.
\end{abstract}

Key words: diet, morphology, Liolaemus elongates, Tropiduridae. 


\section{INTRODUCCIÓN}

Liolaemus Fitzinger, 1843 es un género de lagarto tropidúrido con más de 150 especies (Etheridge 1992) que ocupa todos los nichos posibles en su amplia área austral de génesis y dispersión (Cei 1986). El grupo elongatus sobresale por incluir especies con hábitos esencialmente saxícolas que se distribuyen principalmente en la región andinopatagónica y que estaría en un activo proceso de generación de nuevas especies, como lo evidencia el reconocimiento de nuevas entidades en los últimos años (Cei 1986, Cei \& Ávila 1998).

Liolaemus elongatus es una lagartija muy común y abundante en los roquedales del Noroeste patagónico, habitando desde zonas de bosque húmedo hasta de estepa y desde la alta montaña hasta la meseta (Cei 1986). Su alimentación ha sido descrita como insectívora sobre la base del estudio de una población de alta montaña de la provincia de Mendoza (Videla 1983). Morfológicamente presenta una notoria variación dentro de su extensa área de distribución, desde la provincia de San Juan hasta Santa Cruz (Cei 1974, 1986). Cei (1974) reconoce una tendencia clinal sur-norte en caracteres métricos, merísticos y de coloración basándose en poblaciones distribuidas a lo largo del extremo occidental de las provincias de Chubut, Río Negro, Neuquén y Mendoza. Fundamentalmente, advierte una disminución en el tamaño corporal y un incremento de la palidez de las bandas longitudinales oscuras del dorso (Cei 1974). Dichas poblaciones no fueron analizadas en relación a la dieta, ni fueron descritas las características climático-ambientales de los sitios; sin embargo, las variaciones morfológicas entre poblacionales en especies de lagartijas suelen estar determinadas genéticamente (Guenther et al. 1993, Lamborot \& Eaton 1996) o correlacionadas con variables ecológicas (Hespenheide 1973, Brown \& Thorpe 1991). Un análisis cabal de los patrones de variación geográfica en esta especie es indispensable para poder establecer y discutir las causas de tal variación y para evaluar hipótesis específicas sobre los posibles factores causales. Por consiguiente, el análisis de las variaciones entre poblaciones de $L$. elongatus, geográficamente próximas pero que explotan diferentes ambientes en una misma franja latitudinal, resulta del mayor interés para interpretar el significado de las mismas.

En el noroeste de la Patagonia los autores han reconocido dos poblaciones de L. elongatus que, según observaciones preliminares de campo, aparentemente diferían en su coloración, y que habitan en dos ambientes claramente diferentes, uno de bosque y otro de estepa, distanciados por unos
30 km (Fig. 1). Estudios cromosómicos recientes indican que no existe variación en el cariotipo entre estas poblaciones (Quatrini 1997).

El presente trabajo tiene como objetivo caracterizar la variación en la coloración y el patrón de diseño corporal entre dichas poblaciones, e incorporar otras características morfológicas (caracteres morfométricos más relevantes) y ecológicas (dieta) para examinar las posibles causas de dicha variación.

\section{MATERIALES Y MÉTODOS}

Los sitios donde se realizaron los muestreos representan dos extremos del gradiente climático y vegetacional determinado por la cordillera de los Andes en la provincia de Río Negro, Argentina (Fig. 1), y están caracterizados por conformar un ambiente de estepa y uno de bosque.

Biogeográficamente, el sitio de estepa se encuentra dentro de la Provincia Patagónica (Cabrera \& Willink 1973); es un afloramiento rocoso de 995 m de altitud, ubicado sobre la Ruta Nacional 23 ( $41^{\circ} 05^{\prime} 30^{\prime \prime}$ S, $71^{\circ} 07^{\prime} 30^{\prime \prime}$ O), en la Estancia San Ramón. Se caracteriza por la gran cantidad de lajas de tonalidad rosa tapizadas con líquenes negro-grisáceos y verdes claros. La vegetación periférica al roquedal es típica de la estepa herbácea y arbustiva enana, destacándose la presencia de arbustos medianos y bajos, como Fabiana imbricata Ruiz \& Pav. y Mulinum spinosum (Cav.) Pers., acompañados por hierbas y gramíneas de Mulinum microphyllum (Cav.) Pers., Senecio sericonitens Speg., Ephedra andina Poepp.ex.C.A.Mey, Berberis buxifolia Lam., y Arhenatherum elatius (L.) P. Beauv. Ex J. Presl. En lo alto del roquedal hay parches aislados de vegetación baja y árboles dispersos de Austrocedrus chilensis (D. Don) Florin \& Boutelje. El clima característico de este ambiente es seco con escaso régimen pluvial $(540 \mathrm{~mm}$ año1, Buamscha 1997), templado-frío (aproximadamente $8{ }^{\circ} \mathrm{C}$ de temperatura media anual, según registros de la Estación Meteorológica del Aeropuerto de San Carlos de Bariloche, período 19811990), con ráfagas de viento muy fuertes provenientes del oeste especialmente entre los meses de octubre y marzo (Muñoz \& Garay 1985).

El sitio de bosque pertenece biogeográficamente a la Provincia Subantártica, caracterizada por una vegetación arbórea pluriestratificada, con predominio de Notophagus (Cabrera \& Willink 1973). Es un roquedal ubicado en la ladera oeste del

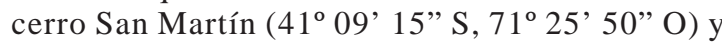
a una altitud de $1.200 \mathrm{~m}$. Su composición litológica es de lavas andesíticas donde dominan las 
coloraciones beige y verde gastado. La vegetación circundante corresponde a la de bosque de transición caracterizado por la presencia de Notophagus antarctica (G. Forst.) Oerst, Austrocedrus chilensis, varios tipos de arbustos como Fabiana imbricata y Mutisia spinosa Ruiz \& Pav., y muchas gramíneas. En esta zona el clima también es templado-frío, pero con temperaturas medias anuales menores que en la estepa debido al fuerte gradiente térmico altitudinal (3 ${ }^{\circ} \mathrm{C}$ a $2.000 \mathrm{~m}$, Mermoz \& Martin 1987). Las precipitaciones son mayores que en el sitio de estepa y alcanzan los $1.400 \mathrm{~mm}^{\text {año-1 }}$ (Buamscha $^{-1}$ 1997).

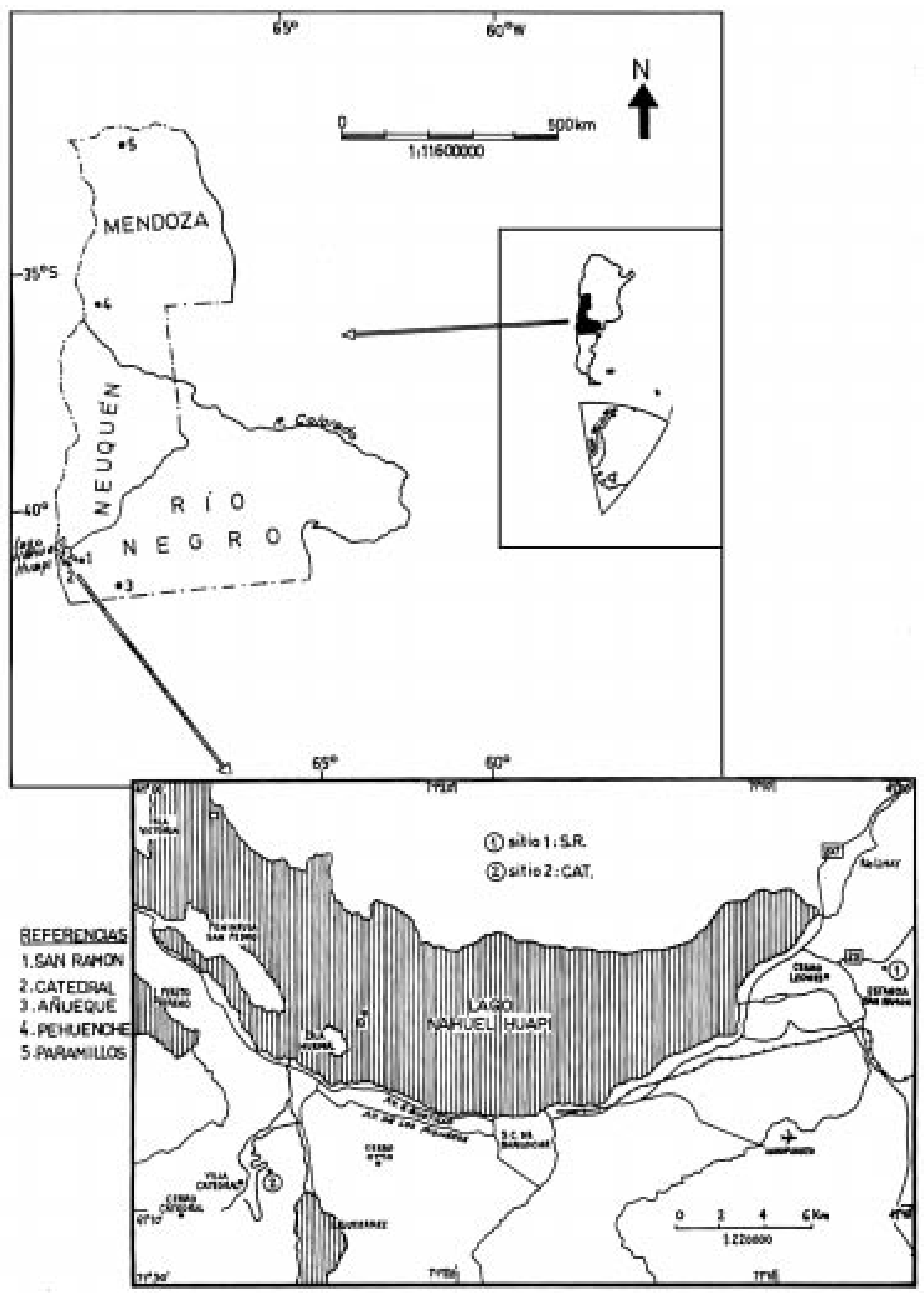

Fig. 1: Ubicación de las localidades citadas en el texto.

Localities cited in the text. 
Los especímenes fueron recolectados entre los meses de diciembre de 1996 y abril de 1997. Los métodos de captura en una y otra población difirieron debido al desigual comportamiento de los individuos. En la estepa se recurrió a trampas pozo pues los ejemplares se mostraron tímidos, esquivos, con una reacción de huída muy veloz y escaso abandono de sus refugios. En el bosque los ejemplares fueron recolectados mediante el uso de lazos corredizos en el extremo de una varilla. En este caso los individuos se mostraron curiosos, muy activos, salían de sus refugios continuamente y pasaban largo tiempo expuestos sobre las rocas.

Se capturaron un total de 47 ejemplares adultos, 22 en el sitio de estepa (13 hembras y nueve machos) y 25 en el sitio de bosque (11 hembras y 14 machos). La variabilidad inherente a la subpoblación de juveniles en lagartijas oscurece las tendencias geográficas de variación en las poblaciones adultas debidas a selección natural. En consecuencia, no se muestrearon juveniles en el presente trabajo, utilizándose como criterio para la separación de clases de edad la longitud hocico-cloaca $(\mathrm{LHC}=65,44)^{1}$.

Los especímenes fueron sacrificados por sobredosis de anestesia (Cloratone-Vetanarcol) entre 24-48 h después de su captura luego de ser inyectados con Colchicina para su análisis citogenético. Cada espécimen fue sexado durante la disección y se le extrajo el contenido estomacal e intestinal, los que fueron conservados en alcohol $70 \%$. Debido al tiempo transcurrido entre la captura y el sacrificio de los ejemplares, el contenido digestivo se conservó muy procesado y no pudieron tomarse medidas de las presas. Posteriormente, los ejemplares fueron fijados en formol al $10 \%$ por $48 \mathrm{~h}$, lavados y conservados en etanol al $70 \%$. Los ejemplares se encuentran depositados en el Departamento de Biología de la Universidad Nacional del Comahue (Anexo 1).

El análisis de variación en la coloración y el patrón de diseño corporal fue realizado mediante observación directa con anterioridad a la fijación de los ejemplares, y considerando los caracteres señalados por Cei (1974). Las variables morfométricas relevadas fueron la longitud hocico-cloaca (desde el margen anterior de la escama mental hasta la abertura cloacal), la longitud de la cabeza (desde el margen anterior de la escama rostral hasta el margen posterior de las últimas escamas cefálicas), el ancho de la cabeza (ancho transversal entre las aberturas auditivas) y la lon-

\footnotetext{
${ }^{1}$ IBARGÜENGOYTÍA N \& V CUSSAC (1996) Actas del Cuarto Congreso Latinoamericano de Herpetología.
}

gitud del maxilar (desde el margen anterior de la escama rostral hasta la comisura bucal). Las mediciones se realizaron con regla aproximando al milímetro y con un pie de metro digital (con una precisión de $0,1 \mathrm{~mm}$ ). Las mediciones bilaterales fueron promediadas para trabajar con un valor único de cada variable.

En el análisis estadístico de los datos métricos se efectuó en primer término un análisis de correlación entre las variables cefálicas y la longitud hocico-cloaca utilizando los coeficientes de Pearson y de Spearman. Debido a la asociación existente demostrada por ambos métodos, los datos se estandarizaron según la siguiente ecuación (Reichow et al. 1991):

$$
\begin{aligned}
& \mathrm{Vt}=\log _{10} \mathrm{Vo}-\left\{\mathrm{CR} \times\left[\log _{10} \mathrm{LHCo}-\text { media }_{\text {sexo }}\right.\right. \\
& \left.\left.\left(\log _{10} \mathrm{~L} \mathrm{mHC}\right)\right]\right\}
\end{aligned}
$$

donde: $\mathrm{Vt}=$ valor de la variable corregida, $\mathrm{Vo}=$ valor de la variable original, $\mathrm{CR}=$ coeficiente de regresión, $\log _{10} \mathrm{LHCo}=\log$ aritmo en base diez de la longitud hocico-cloaca del individuo al cual pertenece la variable a transformar, y media ${ }_{\text {sexo }}$ $\left(\log _{10}\right.$ LHC $)=$ promedio del logaritmo en base diez de la longitud hocico-cloaca de los individuos del sexo al cual pertenece el individuo cuya variable se está transformando.

Esta aproximación logra reducir la correlación entre variables métricas y corregir efectos alométricos, así como también la diferenciación en cuanto a tallas entre las poblaciones bajo análisis. En los casos en que las pendientes de las rectas de ajuste trazadas para el logaritmo de cada variable versus el logaritmo de LHC no fueran homogéneas para los dos sexos, se realizaron ajustes independientes del LHC para cada sexo a fin de transformar las variables correspondientes. Se repitió el análisis de correlación entre pares de variables transformadas con el fin de verificar la efectividad del método en la reducción de la mencionada asociación.

Dado que la isometría o la alometría de una variable dada se ve reflejada en el coeficiente de regresión lineal de su logaritmo versus el logaritmo del tamaño corporal, se calcularon los respectivos coeficientes de regresión (CR) y los intervalos de confianza del $95 \%$ (IC $95 \%$ ) para las variables morfométricas incluidas en este trabajo. Se analizaron previamente los gráficos de dispersión de los datos transformados a logaritmo en base diez con objeto de corroborar la homogeneidad entre las pendientes de las rectas correspondientes a cada sexo entre poblaciones para las distintas variables. Por consiguiente, los datos de ambas poblaciones fueron agrupados indepen- 
dientemente para cada sexo. El cambio exhibido por un carácter dado se consideró isométrico si el IC (95\%) alrededor de su pendiente de regresión incluía a la unidad. Cuando el límite inferior de dicho intervalo estuvo por encima de uno, se consideró al crecimiento como alométrico positivo y cuando el límite superior fue inferior a uno, como alométrico negativo.

Para evaluar la existencia de diferencias significativas en los valores promedios de cada una de las variables métricas registradas, entre sexos y entre poblaciones, se siguió un diseño completamente aleatorizado con un arreglo factorial de $2 \mathrm{x}$ 2 (análisis de varianza), con sexo y sitio como factores. Al existir interacción entre factores se presentan los niveles de significancia de la apertura de tales interacciones. Los supuestos de normalidad y homogeneidad de varianzas fueron evaluados mediante pruebas de bondad de ajuste de Kolmogorov-Smirnov y pruebas de homogeneidad de varianza de Bartlett-Box. El análisis estadístico de los datos se realizó mediante el programa de Estadística Avanzada SPSS para PC.

Los contenidos digestivos fueron observados con microscopio estereoscópico con un aumento de $6,4 \mathrm{x}$; en los casos en que fue necesario se utilizó un aumento de 16 x. La determinación de

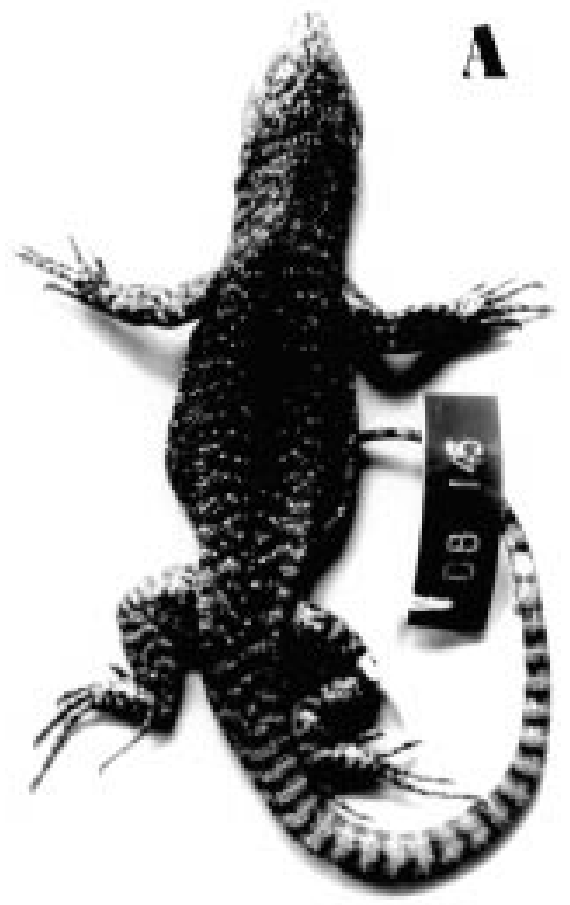

las presas se realizó mediante la identificación de piezas diagnósticas o de cabezas, estableciéndose 12 categorías alimenticias. Las identificaciones realizadas hasta el nivel de orden y, en algunos casos, de familia, se basaron en Bland \& Jacques (1978). El análisis cuantitativo de la dieta incluyó la determinación del número (numerosidad) y frecuencia de aparición de cada categoría alimenticia. La numerosidad correspondió al porcentaje numérico de cada tipo de alimento, mientras que frecuencia a la frecuencia relativa de aparición de cada categoría expresada como porcentaje (Pinkas et al. 1971). Los estómagos vacíos no fueron incluidos en los cálculos de frecuencia.

\section{RESULTADOS}

El análisis cualitativo de la coloración permitió detectar diferencias entre las poblaciones analizadas (Fig. 2). Los individuos de estepa se caracterizaron por presentar una banda vertebral oscura y ancha, con bordes dentados que se anastomosan con los bordes dentados de las bandas longitudinales laterales, también oscuras y anchas, interrumpiendo las bandas claras paravertebrales. La cabeza presentó manchas ne-

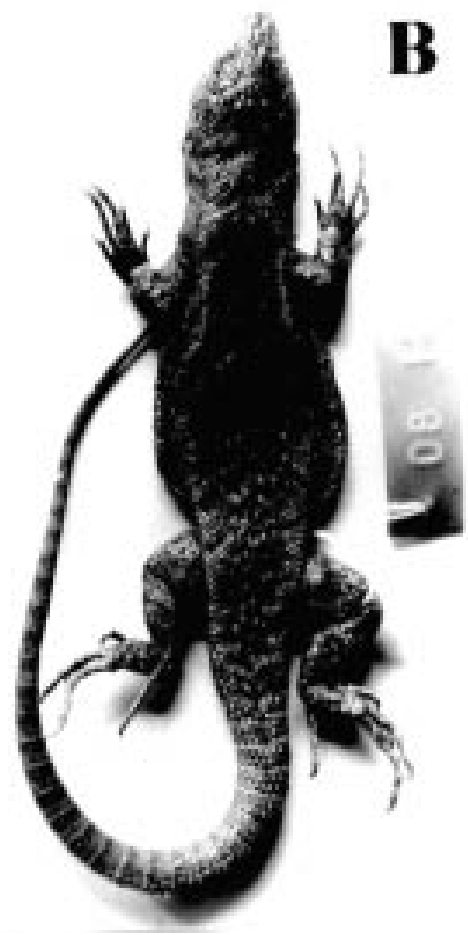

Fig. 2: Ejemplares de L. elongatus de las poblaciones de estepa (A: DB 145) y bosque (B: DB 126). Se indican los rasgos del diseño corporal: bpv = bandas paravertebrales; bv = banda vertebral; $\mathrm{bl}=\mathrm{banda}$ longitudinal lateral; ac = anillado caudal.

Specimens of L. elongatus from the steppe (A: DB 145) and forest populations (B: DB 126). Body traits are as follows: bpv $=$ paravertebral bands; $\mathrm{bv}=$ vertebral band; $\mathrm{bl}=$ lateral longitudinal band; $\mathrm{ac}=$ tail rings. 
gras que formaron un disco de bordes irregulares; los miembros se caracterizaron por tener manchas negras en forma de rayas, mientras que la cola presentó un anillado definido; el vientre presentó pequeñas manchas.

Los individuos de bosque mostraron un diseño diferente. La banda vertebral es más angosta y tenue y sus bordes son poco dentados; las bandas longitudinales laterales son de color más pálido, más angostas y fragmentadas, sin formar anastomosis con los bordes de la banda vertebral; las bandas paravertebrales claras no están interrumpidas. La cabeza tiene manchas poco oscuras esparcidas sin formar un disco. Los miembros presentaron pequeñas manchas poco oscuras sin formar rayas. La cola presentó un anillado desdibujado, poco definido, y el vientre no mostró manchas.

Al considerar la muestra total de individuos se detectó una correlación positiva entre las medidas morfométricas y el tamaño del cuerpo. Una transformación de estas medidas mediante la ecuación de Reichow et al. (1991) logró disminuir los valores de correlación, haciéndolas independientes del tamaño corporal. Los valores de correlación entre cada variable original y entre cada variable transformada y la longitud hocico-cloaca, respectivamente, se presentan en la Tabla 1. Las medidas de tendencia central y de dispersión de todas las variables morfométricas originales y transformadas se muestran en la Tabla 2. Las variables transformadas fueron empleadas en el análisis estadístico subsiguiente. Los datos morfométricos transformados a logaritmos revelaron la existencia de una relación alométrica diferente entre individuos de ambos sexos para cada variable cefálica (Tabla 3). El intervalo de confianza del $95 \%$ para las pendientes de regresión de machos y hembras indicó que en las hembras la cabeza crece en longitud más lentamente que el cuerpo mientras que en los machos la cabeza crece isométricamente. En ambos sexos el crecimiento del ancho de la cabeza fue isométrico con el cuerpo, mientras que la longitud del maxilar mostró un crecimiento alométrico negativo.

Las poblaciones estudiadas se diferenciaron en el tamaño corporal promedio de los individuos, siendo más grandes aquellos de zonas de estepa (factor sitio $\left.\mathrm{F}_{(1,32)}=4,81, \mathrm{P}<0,05\right)$. No se detectaron diferencias significativas entre sexos (factor sexo $\left.\mathrm{F}_{(1,32)}=3,25, \mathrm{P}>0,05\right)$, ni interacción entre sexo y sitio para esta variable (interacción $\left.\mathrm{F}_{(1,32)}=0,00, \mathrm{P}>0,05\right)$.

En el caso de las variables cefálicas se registró una interacción entre los factores sitio y sexo. Por lo tanto, se determinó la variación existente en las variables cefálicas entre sexos en cada una de las poblaciones, así como también la variación entre poblaciones para individuos de cada sexo. En la estepa los machos mostraron mayores valores que las hembras sólo en el ancho de la cabeza (factor sexo-estepa $\left.\mathrm{F}_{(1,32)}=7,29, \mathrm{P}<0,05\right)$, mientras que en el bosque los machos superaron a las hembras en el valor de todos los parámetros cefálicos (factor sexo-bosque: longitud de la cabeza $\mathrm{F}_{(1,32)}=$ $15,79, \mathrm{P}<0,05$; ancho de la cabeza $\mathrm{F}_{(1,32)}=20,79$, $\mathrm{P}<0,05$; longitud del maxilar $\mathrm{F}_{(1,32)}=5,47, \mathrm{P}<$ $0,05)$. Los machos en ambas poblaciones difirieron en la longitud de la cabeza (factor sitiomachos $\left.\mathrm{F}_{(1,32)}=5,38, \mathrm{P}<0.05\right) \mathrm{y}$ en la longitud del maxilar (factor sitio-machos $\mathrm{F}_{(1,32)}=9,39, \mathrm{P}<$ $0,05)$. Los individuos de bosque presentaron mayores valores relativos para estas variables que

\section{TABLA 1}

Coeficientes de correlación entre la longitud hocico-cloaca (LHC), las variables métricas originales y las variables métricas transformadas. $\mathrm{LCA}=$ longitud de la cabeza, ACA = ancho de la cabeza, LMX = longitud del maxilar. Valor superior: coeficiente de Pearson; valor inferior: coeficiente de Spearman. Se indica entre paréntesis el nivel de significancia

Coefficients of correlation between LHC and the original metric variables and the transformed metric variables respectively. Value above is the Pearson coefficient. Value bellow is the Spearman coefficient. Significance level is indicated within parenthesis

Muestra completa $(\mathrm{n}=47)$

\begin{tabular}{ccc} 
Variables & Original & Transformada \\
\hline LCA vs LHC & $0,809(\mathrm{P}<0,05)$ & $0,105(\mathrm{P}<0,05)$ \\
ACA vs LHC & $0,774(\mathrm{P}<0,05)$ & $0,053(\mathrm{P}<0,05)$ \\
& $0,803(\mathrm{P}<0,05)$ & $0,087(\mathrm{P}<0,05)$ \\
LMX vs LHC & $0,756(\mathrm{P}<0,05)$ & $0,096(\mathrm{P}<0,05)$ \\
& $0,499(\mathrm{P}<0,05)$ & $0,025(\mathrm{P}<0,05)$ \\
& $0,527(\mathrm{P}<0,05)$ & $-0,029(\mathrm{P}<0,05)$ \\
\hline
\end{tabular}


TABLA 2

Variables morfométricas originales y transformadas y sus estadígrafos para los individuos de L. elongatus discriminados por sexo y sitio. Los valores superiores corresponden a la media \pm desviación estándar; los valores inferiores corresponden al rango

Morphometric and transformed variables and their descriptive statistics for L. elongatus individuals classified by sex and site. The upper values represent the mean \pm standard deviation, and the lower values represent the range

\begin{tabular}{|c|c|c|c|c|}
\hline \multirow[t]{2}{*}{ Variable } & \multicolumn{2}{|c|}{ Estepa } & \multicolumn{2}{|c|}{ Bosque } \\
\hline & $\begin{array}{c}\text { Machos } \\
(\mathrm{n}=9)\end{array}$ & $\begin{array}{c}\text { Hembras } \\
(\mathrm{n}=13)\end{array}$ & $\begin{array}{l}\text { Machos } \\
(\mathrm{n}=14)\end{array}$ & $\begin{array}{c}\text { Hembras } \\
(\mathrm{n}=11)\end{array}$ \\
\hline LHC & $\begin{array}{r}77,77 \pm 6,24 \\
69,00-86,00\end{array}$ & $\begin{array}{r}75,46 \pm 3,43 \\
69,00-80,00\end{array}$ & $\begin{array}{r}73,50 \pm 3,83 \\
67,00-83,00\end{array}$ & $\begin{array}{r}71,27 \pm 2,83 \\
67,00-75,00\end{array}$ \\
\hline LCA & $\begin{array}{r}16,37 \pm 1,59 \\
13,05-18,29\end{array}$ & $\begin{array}{r}15,96 \pm 0,63 \\
14,83-16,85\end{array}$ & $\begin{array}{r}16,26 \pm 0,63 \\
15,55-17,69\end{array}$ & $\begin{array}{r}15,13 \pm 0,48 \\
14,36-16,09\end{array}$ \\
\hline ACA & $\begin{array}{r}14,18 \pm 1,84 \\
11,71-16,66\end{array}$ & $\begin{array}{r}13,74 \pm 0,65 \\
12,65-14,72\end{array}$ & $\begin{array}{r}13,52 \pm 0,83 \\
12,56-15,20\end{array}$ & $\begin{array}{r}12,59 \pm 0,21 \\
12,28-12,97\end{array}$ \\
\hline LMX & $\begin{array}{r}9,94 \pm 0,86 \\
7,89-10,83\end{array}$ & $\begin{array}{l}10,17 \pm 0,46 \\
9,47-10,93\end{array}$ & $\begin{array}{r}10,41 \pm 0,59 \\
9,52-11,42\end{array}$ & $\begin{array}{r}9,82 \pm 0,50 \\
8,51-10,47\end{array}$ \\
\hline $\mathrm{LCA}_{\mathrm{t}}$ & $\begin{array}{r}1,20 \pm 0,01 \\
1,15-1,22\end{array}$ & $\begin{array}{r}1,19 \pm 0,01 \\
1,17-1,22\end{array}$ & $\begin{array}{l}1,22 \pm 0,01 \\
1,20-1,24\end{array}$ & $\begin{array}{l}1,19 \pm 0,01 \\
1,17-1,20\end{array}$ \\
\hline $\mathrm{ACA}_{\mathrm{t}}$ & $\begin{array}{l}1,14 \pm 0,02 \\
1,10-1,16\end{array}$ & $\begin{array}{l}1,13 \pm 0,02 \\
1,09-1,16\end{array}$ & $\begin{array}{l}1,14 \pm 0,01 \\
1,11-1,17\end{array}$ & $\begin{array}{l}1,11 \pm 0,01 \\
1,09-1,12\end{array}$ \\
\hline $\mathrm{LMX}_{\mathrm{t}}$ & $\begin{array}{l}0,99 \pm 0,03 \\
0,91-1,02\end{array}$ & $\begin{array}{r}1,00 \pm 0,0 \\
0,96-1,04\end{array}$ & $\begin{array}{l}11,02 \pm 0,0 \\
0,99-1,05\end{array}$ & $\begin{array}{r}11,00 \pm 0,01 \\
0,95-1,01\end{array}$ \\
\hline
\end{tabular}

los de estepa. Por su parte, las hembras se diferenciaron en el ancho de la cabeza (factor sitiohembras $\left.\mathrm{F}_{(1,32)}=12,11, \mathrm{P}<0,05\right)$; en este caso los ejemplares de estepa excedieron a aquellos de bosque.

En relación a la dieta, los resultados del análisis se presentan en las Tablas 4 y 5. Los estómagos sin ningún contenido fueron dos en la población de estepa y uno en el bosque. Desde el punto de vista cualitativo no se detectó diferencias entre pobla- ciones. En cuanto a la numerosidad, los formícidos contribuyeron en ambas poblaciones con más del $50 \%$, pero el segundo tipo de presa en importancia difirió; en la población de estepa correspondieron a insectos homópteros, mientras que en el bosque a semillas. En ambas poblaciones el tercer tipo de presa en orden de importancia correspondió a insectos himenópteros no formícidos. Con respecto a la frecuencia de ocurrencia de las distintas presas, los formícidos fueron mayoritarios en ambas po-

\section{TABLA 3}

Resultados del análisis de regresión entre las variables morfométricas originales (transformadas a logaritmo) y el logaritmo del LHC; b = pendiente de regresión; ES = error estándar; IC = intervalo de confianza del $95 \% ; \mathrm{P}=$ significancia estadística de la regresión

Results of regression analysis between the original morphometric variables and the logarithm of LHC; b = regression slope; $\mathrm{ES}=$ standard error, $\mathrm{IC}=95 \%$ confidence interval $; \mathrm{P}=$ statistical significance of the regression

\begin{tabular}{|c|c|c|c|c|c|c|}
\hline Variable & Sexo & $\begin{array}{c}\text { Ordenada } \\
\text { al origen }\end{array}$ & $b \pm E S b$ & IC $95 \%$ & $\mathrm{P}$ & Alometría \\
\hline LCA & $\begin{array}{l}0^{7} \\
0\end{array}$ & $\begin{array}{l}-0,320 \\
-0,022\end{array}$ & $\begin{array}{l}0,816 \pm 0,121 \\
0,650 \pm 0,123\end{array}$ & $\begin{array}{l}0,563-1,069 \\
0,394-0,907\end{array}$ & $\begin{array}{l}<0,05 \\
<0,05\end{array}$ & $\begin{array}{l}\text { Isométrico } \\
\text { Alométrico (-) }\end{array}$ \\
\hline ACA & $\begin{array}{l}\sigma^{7} \\
0\end{array}$ & $\begin{array}{l}-1,180 \\
-0,069\end{array}$ & $\begin{array}{l}1,236 \pm 0,137 \\
0,637 \pm 0,194\end{array}$ & $\begin{array}{l}0,950-1,521 \\
0,235-1,040\end{array}$ & $\begin{array}{l}<0,05 \\
<0,05\end{array}$ & $\begin{array}{l}\text { Isométrico } \\
\text { Isométrico }\end{array}$ \\
\hline LMX & $\begin{array}{l}+ \\
0^{7} \\
\stackrel{9}{+}\end{array}$ & $\begin{array}{r}0,099 \\
-0,070\end{array}$ & $\begin{array}{l}0,484 \pm 0,216 \\
0,573 \pm 0,177\end{array}$ & $\begin{array}{l}0,035-0,934 \\
0,204-0,942\end{array}$ & $\begin{array}{l}<0,05 \\
<0,05\end{array}$ & $\begin{array}{l}\text { Alométrico (-) } \\
\text { Alométrico (-) }\end{array}$ \\
\hline
\end{tabular}


TABLA 4

Número y frecuencia en cada población analizada. Valores entre paréntesis: machos/hembras

Number and frequency in each analyzed population. Values between parenthesis: males/females

\begin{tabular}{|c|c|c|c|c|}
\hline \multirow[t]{2}{*}{ Taxón } & \multicolumn{2}{|c|}{ Número } & \multicolumn{2}{|c|}{ Frecuencia (\%) } \\
\hline & Estepa & Bosque & Estepa & Bosque \\
\hline Formicidae & $304(71 / 233)$ & $300(186 / 114)$ & $60,56(35,32 / 77,41)$ & $53,67(74,70 / 36,77)$ \\
\hline Hymenoptera no FO & $65(31-34)$ & $40(10 / 30)$ & $12,95(15,42 / 11,30)$ & $7,16(4,02 / 9,68)$ \\
\hline Semillas & $27(14 / 13)$ & $168(37 / 131)$ & $5,38(6,97 / 4,32)$ & $30,05(14,86 / 42,26)$ \\
\hline Homoptera & $75(66 / 9)$ & $29(1 / 28)$ & $14,94(32,84 / 2,99)$ & $5,19(0,40 / 9,03)$ \\
\hline Diptera & $8(1 / 7)$ & $5(2 / 3)$ & $1,59(0,50 / 2,99)$ & $0,89(0,80 / 0,97)$ \\
\hline Larvas & 0 & $2(1 / 1)$ & 0,00 & $0,36(0,40 / 0,32)$ \\
\hline Hemiptera & $1(1 / 0)$ & 0 & $0,20(0,50 / 0)$ & 0,00 \\
\hline Curculionidae & $4(2 / 2)$ & $2(2 / 0)$ & $0,80(1 / 0,66)$ & $0,36(0,80 / 0,00)$ \\
\hline Crisomelidae & $7(5 / 2)$ & $7(6 / 1)$ & $1,39(2,49 / 0,66)$ & $1,25(2,41 / 0,32)$ \\
\hline Carabidae & $9(9 / 0)$ & $4(3 / 1)$ & $1,79(4,48 / 0,00)$ & $0,72(1,20 / 0,32)$ \\
\hline Lepidoptera & $1(1 / 0)$ & $1(0 / 1)$ & $0,20(0,50 / 0,00)$ & $0,18(0,40 / 0,00)$ \\
\hline Acarae & $1(0 / 1)$ & $1(1 / 0)$ & $0,20(0,00 / 0,50)$ & $0,18(0,40 / 0,00)$ \\
\hline Restos foliares & $\mathrm{X}$ & $\mathrm{X}$ & $\mathrm{X}$ & $\mathrm{X}$ \\
\hline Total & $502(201 / 301)$ & $559(249 / 310)$ & 100 & 100 \\
\hline
\end{tabular}

blaciones con más del $85 \%$, seguidos por himenópteros no formícidos con más del $50 \%$. La tercera presa más frecuente en ambientes de estepa fueron los homópteros, mientras que en el bosque el tercer tipo de presa más frecuente fueron semillas. En las Tablas 4 y 5 se destacan entre paréntesis los valores de numerosidad y frecuencia considerando los sexos por separado.

\section{DISCUSIÓN}

Las poblaciones estudiadas se diferencian en la coloración y en el tamaño corporal promedio, no existiendo dimorfismo sexual en estos aspectos.

En términos generales se observa una coloración corporal más oscura y un mayor contraste entre el patrón de diseño y la coloración de fondo

TABLA 5

Frecuencia (\%) de presas en las poblaciones analizadas. Valores entre paréntesis: machos/hembras

Frequency of prey items in each analyzed population. Values between parenthesis: males/females

\begin{tabular}{lrr}
\hline Taxón & Estepa $(\mathrm{n}=20)$ & Bosque $(\mathrm{n}=24)$ \\
\hline Formicidae & $95,00(88,89 / 84,62)$ & $87,50(92,31 / 81,82)$ \\
Hymenoptera no FO & $75,00(88,89 / 53,85)$ & $50,00(38,46 / 63,64)$ \\
Semillas & $25,00(11,11 / 30,77)$ & $37,50(30,77 / 45,45)$ \\
Homoptera & $40,00(55,56 / 23,08)$ & $16,67(7,69 / 27,27)$ \\
Diptera & $25,00(11,11 / 30,77)$ & $20,83(15,38 / 27,27)$ \\
Larvas & $5,00(0,50 / 0)$ & $8,33(15,38 / 0,00)$ \\
Hemiptera & $20,00(22,22 / 15,38)$ & 0,00 \\
Curculionidae & $30,00(44,44 / 15,38)$ & $8,33(15,38 / 0,00)$ \\
Crisomelidae & $15,00(33,33 / 0,00)$ & $20,83(30,77 / 9,09)$ \\
Carabidae & $5,00(11,11 / 0,00)$ & $12,50(15,38 / 9,09)$ \\
Lepidoptera & $5,00(0,00 / 7,69)$ & $4,17(0,00 / 9,09)$ \\
Acarae & $25,00(33,33 / 18,18)$ & $4,17(7,69 / 0,00)$ \\
Restos foliares & & $33,33(30,76 / 36,36)$ \\
\hline
\end{tabular}


en los individuos de la estepa que en el bosque, esto último es especialmente notable en el anillado de la cola (Fig. 2). Esta variación ha sido registrada anteriormente en sentido sur-norte a lo largo de las provincias de Chubut, Río Negro, Neuquén y Mendoza, y representaría los dos extremos de la tendencia clinal en la coloración de L. elongatus descrita por Cei (1974). En nuestro estudio los mismos extremos de variación se observan en sentido este-oeste y entre poblaciones distanciadas por sólo $30 \mathrm{~km}$. Las características de la coloración de los individuos de estepa y bosque recuerdan, respectivamente, a la de los individuos de Añueque (Río Negro), y Pehuenche y Paramillos (Mendoza) (Fig. 1) (Cei 1974). Debido a que las poblaciones analizadas se encuentran aproximadamente a la misma latitud, la tendencia descrita por Cei (1974) no representaría una variación en una sola dimensión espacial. Esto se ve corroborado por la existencia del patrón de coloración descrito para la población de estepa en ejemplares de otra población que se encuentra ubicada unos $60 \mathrm{~km}$ al Este de la misma, a una latitud y altitud comparable (observaciones personales). Esta variación podría responder a factores genéticos (deriva génica) o selectivos (divergencia adaptativa o selección sexual). Considerando la proximidad geográfica entre las poblaciones analizadas en este trabajo, un modelo vicariante es el que mejor explicaría la divergencia encontrada en los patrones de coloración y diseño.

De acuerdo a los análisis efectuados por Quatrini (1997), no habría una componente cromosómica de esta variación como ocurre en otras especies de Liolaemus (Lamborot \& Eaton 1992). Sin embargo, y teniendo en cuenta la presencia de una barrera geográfica como es el río Ñirihuau (Fig. 1), la capacidad limitada de dispersión de los individuos y la distribución muy discontinua de los roquedales en los que habitan, además de las importantes diferencias ambientales entre los sitios, es posible suponer la ausencia de intercambio génico entre ellas. Por consiguiente, se advierte como necesaria la cuantificación de la variación genética entre las poblaciones a través de técnicas más sensibles.

Es interesante que la coloración oscura de los individuos de estepa los hace poco evidentes en los sectores rocosos cubiertos por líquenes donde se asolean, pero los destaca en las zonas utilizadas para el desplazamiento y alimentación por su contraste con el color claro de la tierra y el pedregal suelto. Es probable que las características más abiertas del ambiente de estepa determinen una mayor exposición a depredadores, especialmente aéreos, por parte de individuos de estepa en comparación con aquellos de bosque, donde la vegetación circundante es muy utilizada por los ejemplares para ocultarse y su coloración más clara resulta críptica respecto al sustrato rocoso. Ésto concuerda con diferencias en el comportamiento de los individuos. Los ejemplares de estepa mostraron respuesta de huída más rápidas y fueron más reticentes a salir de sus refugios que aquellos del bosque. Tales patrones etológicos posiblemente hayan sido seleccionados diferencialmente en cada uno de estos ambientes.

Por otra parte, los valores de tamaño corporal promedio son mayores en la población de estepa. Cei (1974) y Bottari (1975) reportan disminución en el tamaño corporal adulto de otras poblaciones de L. elongatus en sentido sur-norte, tendencia que en el caso de las poblaciones aquí analizadas se manifiesta en un gradiente esteoeste. En varias especies de lagartijas el tamaño promedio del cuerpo es menor a altitudes mayores (Malhotra \& Thorpe 1991). El carácter más estacional del clima y el menor período anual disponible para el crecimiento a mayor altitud reducirían las tasas de crecimiento anual (Báez \& Brown 1997). Por consiguiente, la diferencia de altitud entre los sitios de estepa y bosque podría ser la causa de la variación detectada en el tamaño corporal. La tendencia en la variación de tamaño registrada por Cei (1974) podría estar también relacionada con la altitud más que con la latitud. Otra explicación posible a esta variación es que exista una correlación positiva entre el tamaño del lagarto y el tamaño promedio de la presa consumida (largo y/o volumen) como ha sido comprobado en otras especies de Liolaemus (Ortiz \& Riveros 1976, Jaksic \& Fuentes 1980, Vega 1999). Debido a que durante nuestros análisis de dieta no fue posible registrar el tamaño de las presas no es posible examinar esta hipótesis.

No se encontró dimorfismo sexual en las características de coloración y diseño, ni en el tamaño corporal promedio de las poblaciones analizadas aunque sí se observaron variaciones en las medidas cefálicas entre los sexos de cada población. En el bosque, machos y hembras se diferenciaron entre sí en los tres parámetros cefálicos evaluados, mientras que en la estepa los sexos se diferenciaron únicamente en uno de ellos. La existencia de variación geográfica en el dimorfismo sexual de los caracteres cefálicos sugiere que distintos mecanismos selectivos pudieran estar operando en estas poblaciones. Slatkin (1984) indica que diferencias de hábitat son suficientes para producir diferencias en la evolución del dimorfismo sexual debido a facto- 
res ecológicos. También es posible que el mayor número de caracteres diferenciales entre sexos en el bosque se deba a que esta población se encuentra más próxima a su tamaño corporal asintótico, a pesar de su menor tamaño relativo; mientras que el menor grado de diferenciación sexual en la estepa podría ser explicado por un distanciamiento de la muestra respecto de su asíntota poblacional, eventualmente por efecto de una depredación superior, circunstancia que no ha sido evaluada en este trabajo.

Las diferencias en las medidas cefálicas y/o en las del maxilar de los lagartos suelen correlacionarse con el tamaño de las presas (Hespenheide 1973), aunque existen casos en los que no se han encontrado diferencias significativas en el tamaño promedio de presa consumido por los sexos a pesar de sus diferentes parámetros cefálicos (Vega 1999). Debido a que no se analizaron los tamaños de presa consumidos por las poblaciones, no es posible contrastar estas hipótesis, ni establecer si existe un uso diferencial de los recursos (en relación a categorías de tamaño) entre sexos en una y/o otra población.

La composición cualitativa de la dieta indica que L. elongatus de las poblaciones estudiadas tienen una alimentación omnívora. Fundamentalmente consumen formícidos y otros artrópodos, pero también es frecuente la presencia de elementos vegetales en los contenidos digestivos de los individuos de las dos poblaciones demostrando que su ingestión no ha sido accidental, sino que representan una categoría alimenticia más dentro de la dieta. Se considera que las semillas provendrían de frutos pues se han hallado restos vegetales acompañantes semejantes a los cálices de los mismos. Además, en otra población de la especie de las cercanías de San Carlos de Bariloche fue hallado un fruto de considerable tamaño en el estómago de un individuo ${ }^{2}$. También se considera que se han consumido hojas. En otras poblaciones de la especie analizadas recientemente fueron halladas hojas y/o semillas en abundante número ${ }^{2}$ corroborando las apreciaciones vertidas en este trabajo respecto al consumo intencional de elementos vegetales.

Según Pough (1973), la ingestión de vegetales por lagartos está limitada a aquellas especies de tamaño grande y peso superior a $100 \mathrm{~g}$. Sin embargo, el peso promedio de los individuos de $L$. elongatus analizados en este trabajo es de 10,68 g. En otras especies de Liolaemus de dimensiones

${ }^{2}$ ALBINO AM, MM BUNGE \& R QUATRINI (2000)

Décimoquinta reunión de la Asociación de

Herpetología Argentina, resumen 7. menores a los $100 \mathrm{~g}$ también se ha hallado que los vegetales constituyen un ítem alimenticio significativo (Ortiz \& Riveros 1976, Jaksic \& Fuentes 1980, Rocha 1989, Van Sluys 1993, Acosta et al. 1996), hecho que ha sido demostrado experimentalmente (Fuentes \& Di Castri 1975). En algunas especies del género se ha comprobado que los individuos de mayor tamaño consumen una mayor cantidad de elementos vegetales que los de menor tamaño (Jaksic \& Fuentes 1980, Rocha 1998). En el caso de las poblaciones analizadas en este trabajo se encuentra que la población de mayor tamaño promedio (la de estepa) presentan una menor frecuencia de semillas en relación a la población de menor tamaño promedio (la de bosque), contrastando con las opiniones mencionadas.

Si bien las semillas fueron examinadas como una categoría única, el tipo de semilla más frecuente en los individuos de bosque sólo se encontró en un individuo de estepa. Se trata de semillas correspondientes a una Hydrophyllacea, nativa común en bosques de ciprés, en zonas rocosas (Ezcurra comunicación personal). El ciprés (Austrocedrus chilensis) es más frecuente en los hábitat de bosque examinados comparado con sitios de estepa (observaciones personales), de modo que aún cuando no se ha evaluado cuantitativamente la disponibilidad de esta Hydrophyllacea en uno y otro ambiente, se infiere que el tipo de fruto más consumido en cada sitio probablemente depende de la abundancia del mismo en cada ambiente.

La presencia de elementos de origen vegetal en la dieta de estas poblaciones de L. elongatus difiere de los resultados expuestos por Videla (1983) para una población de la especie de la región precordillerana de Paramillos, en Mendoza (3.000 m de altitud), donde el componente vegetal es nulo. Esta población se caracteriza por explotar un ambiente de alta montaña, donde el suelo permanece desnudo la mayor parte del año y las condiciones climáticas son extremas, caracterizando la Provincia Fitogeográfica Puneña (Videla 1983). Según el modelo de Jaksic (1978), sería esperable que en ambientes pobres o fluctuantes la transición desde carnivoría a herbivoría se produzca a tamaños relativamente pequeños. Sin embargo, en la localidad estudiada por Videla (1983), que corresponde claramente a un ambiente fluctuante y donde las lagartijas son las de menores dimensiones (LHC: 59-63 \pm 0,64-0,67 en machos y LHC: 56-59 \pm 0,65-0,64 en hembras) (Cei 1974), el consumo vegetal fue nulo, mientras que en las poblaciones analizadas en este trabajo, que también explotan ambientes fluctuantes, existe un significativo consumo de elementos vegetales. 
Por lo tanto, la disponibilidad de elementos vegetales podría ser uno de los factores más importantes que determinen la tendencia a la omnivoría de L. elongatus. Sin embargo, es posible que otras variables intrínsecas a las poblaciones o a las comunidades de reptiles de las que forman parte (competencia intra e interespecífica, variabilidad en parámetros métricos, características conductuales, variaciones morfológicas, histológicas o químicas en el sistema digestivo), además de las variables ambientales que determinan la oferta de alimento (características climáticas y florísticas, condiciones de estacionalidad diferencial), influyan sobre el consumo de elementos de origen vegetal por parte de distintas poblaciones de $L$. elongatus.

Se coincide con los resultados de Videla (1983) en cuanto al mayoritario porcentaje de himenópteros formícidos en la dieta de $L$. elongatus. En relación a estos altos porcentajes es posible decir que varias especies de Liolaemus consumen como item mayoritario en numerosidad, frecuencia y volumen este tipo de alimento; entre ellas, L. darwini y L. monticola (Viana et al. 1994, Fuentes \& Ipinza 1979). Los formícidos representan un recurso habitualmente disponible en gran cantidad que algunas especies de lagartijas explotan utilizando una estrategia de búsqueda activa. El costo energético que esto demanda estaría compensado por la facilidad para atrapar a las presas y la abundancia de las mismas. Otros tipos de alimento serían ocasionales en la dieta de L. elongatus.

Es necesario destacar finalmente que, como ya fuera reconocido por Cei (1979), el grupo elongatus demuestra haber sufrido especiación post-pleistocénica resultando en muchas líneas de especies afines; entre otras, L. petrophilus (Cei \& Avila 1998). Algunas de estas especies se habrían diferenciado como consecuencia de la acción de la deriva génica sobre poblaciones pequeñas aisladas de otras poblaciones del grupo. Al menos éste habría sido el caso de las nuevas especies caracterizadas por su endemismo en la región central de Neuquén y en una pequeña área por encima de los $3.000 \mathrm{~m}$ en el norte de la misma provincia ${ }^{3}$. Las mismas presentan particularidades genéticas, morfológicas y biológicas. En el caso de L. elongatus es claro que los factores diversificadores continúan actuando aunque la naturaleza de los mismos es aún incierta.

\footnotetext{
${ }^{3}$ ÁVILA LJ, M MORANDO \& CHF PÉREZ (2000)

Décimo quinta reunión de la Asociación de

Herpetología Argentina, resumen 10.
}

\section{CONCLUSIONES}

Las poblaciones analizadas se diferencian en la coloración y diseño corporal, y en el tamaño corporal promedio. Lo primero podría estar relacionado con fenómenos genéticos o selectivos, mientras que lo segundo dependería de la altitud a la que se encuentra la población o al tamaño promedio de presas que consumen. No se detectó dimorfismo sexual en estos caracteres pero sí en los parámetros cefálicos, aunque el mismo se manifiesta de manera diferente en cada población, posiblemente por la acción de distintos mecanismos selectivos en cada ambiente. La dieta de los L. elongatus de estas poblaciones resulta ser omnívora en contraste con una población de alta montaña donde no se describieron componentes vegetales. Sin embargo, en las tres poblaciones hay coincidencia respecto al mayoritario consumo de formícidos.

Las variaciones morfológicas y ecológicas reconocidas entre las dos poblaciones analizadas indican que las poblaciones de L. elongatus a lo largo de su extensa área de distribución se encuentran sujetas a procesos de diversificación donde los factores responsables aún son inciertos.

\section{AGRADECIMIENTOS}

A Miguel Christie por sus sugerencias respecto a sitios de muestreo. A Horacio Planas, Javier Puntieri y Jorge Chiapella por su colaboración en la caracterización geológica y florística de los sitios. A Florencia Funes por su ayuda en las tareas de campo. A Nora Baccalá por el asesoramiento para los análisis estadísticos. A Jorge Fariña por su colaboración para la determinación de los artrópodos de la dieta. A Laura Vega por la lectura crítica de un primer manuscrito. A dos revisores anónimos y al editor asociado de la revista por su inestimable aporte para mejorar el manuscrito. El presenta trabajo formó parte del Proyecto de Investigación CRUB 04/B076 de la Universidad Nacional del Comahue.

\section{LITERATURA CITADA}

ACOSTA JC, LJ ÁVILA \& G BLANCO (1996) Ecología de Liolaemus boulengeri (Sauria: Tropiduridae) en el Noroeste de la estepa patagónica (Ing. Jacobacci, Río Negro, Argentina). Cuadernos de Herpetología 9: 100-107. 
BÁEZ M \& RP BROWN (1997) Testing multivariate patterns of within-island differentiation in Podacris dugesii from Madeira. Journal of Evolutionary Biology 10: 575-587.

BLAND RG \& HE JACQUES (1978) How to know the insects. W. C. Brown Company Publishers, Dubuque, Iowa. 409 pp.

BOTTARI CV (1975) Poblaciones altitudinarias aisladas de Liolaemus elongatus del Volcán Payún Liso. Relaciones taxoserológicas y biogeográficas en el conjunto elongatus-austromendocinus del sureste árido de Mendoza. Deserta (Argentina) 4: 185-194.

BROWN RP \& RS THORPE (1991) Within-island microgeographic variation in the colour pattern of the skink, Chalcides sexlineatus: pattern and cause. Journal of Evolutionary Biology 4: 557-574.

BUAMSCHA MG (1997) Indicadores de economía de nitrógeno en Austrocedrus chilensis a lo largo de un gradiente de humedad. Tesis de Licenciatura, Centro Regional Universitario Bariloche, Universidad Nacional del Comahue, Argentina. 113pp.

CABRERA AL \& A WILLINK (1973) Biogeografía de América Latina. Monografía de la Secretaría General de la Organización de Estados Americanos, Washington, District of Columbia. vi +120 pp.

CEI JM (1974) Revision of the Patagonian iguanids of the Liolaemus elongatus complex. Journal of Herpetology 8: 219-229.

CEI JM (1979) The Patagonian herpetofauna. En: Duellman WE (ed) The South American Herpetofauna: its origin, evolution and dispersal. Monograph of the Museum of Natural History, University of Kansas 7: 309-337.

CEI JM (1986) Reptiles del centro, centro-oeste y sur de Argentina. Herpetofauna de las zonas áridas y semiáridas. Monografía del Museo Regionale di Scienze Naturali. 527 pp.

CEI JM \& LJ Á VILA (1998) Reconocimiento de la categoría de especie para Liolaemus petrophilus (Squamata, Tropiduridae, Liolaeminae). Facena 14: 80.

ETHERIDGE R (1992) A new psammophilus lizard of the genus Liolaemus (Squamata: Tropiduridae) from north-western Argentina. Bolletino dil Museo Regionale di Scienze Naturali di Torino (Italy) 10: 119.

FUENTES ER \& F DI CASTRI (1975) Ensayo de herbivoría experimental en especies de Liolaemus (Iguanidae) chilenos. Anales del Museo de Historia Natural de Valparaíso (Chile) 8: 66-75.

FUENTES ER \& J IPINZA (1978) A note on the diet of Liolaemus monticola (Reptilia, Lacertilia, Iguanidae) Journal of Herpetology 13: 123-124.

GUENTHER MM, GW FERGUSON, HL SNELL \& H SENLL (1993) The variation and genetic basis of dorsal color pattern in the desert side-blotched lizard, Uta stansburiana stejnegeri. Journal of Herpetology 27: 199-205.

HESPENHEIDE HA (1973) Ecological inferences from morphological data. Annual Review of Ecology and Systematics 4: 213-229.

JAKSIC FM (1978) ¿A qué tamaño se hace herbívora una lagartija? Anales del Museo de Historia Natural de Valparaíso (Chile) 11: 113-115.
JAKSIC FM \& ER FUENTES (1980) Observaciones autoecológicas en Liolaemus nitidus (Lacertilia: Iguanidae). Studies on Neotropical Fauna and Environment 15: 109-124.

LAMBOROT M \& LC EATON (1992) Concordance of morphological variation and chromosomal races in Liolaemus monticola (Tropiduridae) separated by riverine barriers in the Andes. Zeitschrift für Zoologische Systematik und Evolutionsforchung 30: 189-200.

MALHORTRA A \& RS THORPE (1991) Microgeographic variation in Anolis oculatus, on the island Dominica, West Indies. Journal of Evolutionary Biology 4: 321335.

MERMOZ M \& C MARTIN (1987) Mapa de vegetación del Parque y la Reserva Nacional Nahuel Huapi. Secretaría de Ciencia y Técnica de la Nación, Delegación Regional de la Patagonia, San Carlos de Bariloche, Argentina. 22 pp.

MUÑOZ E \& A GARAY (1985) Caracterización climática de la Provincia de Río Negro. Estación Experimental del Instituto Nacional de Tecnología Agropecuaria, Región Agropecuaria de San Carlos de Bariloche, Argentina. $56 \mathrm{pp}$.

ORTÍZ JC \& GM RIVEROS (1976) Hábitos alimenticios de Liolaemus nicromaculatus kuhlmani, Müller y Hellmich. Anales del Museo de Historia Natural de Valparaíso (Chile) 9: 131-140.

PINKAS L, M OLIPHANAT \& Z IVERSON (1971) Food habits of albacore bluefin tuna and bonito in California waters. Department of Fish and Games Fish Bulletin 152: 1-105.

POUGH FA (1973) The lizard energetics and diet. Ecology 54: $837-844$

QUATRINI R (1997) Comparación morfológica y citogenética entre dos poblaciones de Liolaemus elongatus (Reptilia: Squamata: Tropiduridae) del Noroeste Patagónico. Tesis de Licenciatura, Centro Regional Universitario Bariloche, Universidad Nacional del Comahue, Argentina. 132 pp.

REICHOW D, C LARGIADER, CH KLIGERBERG, C CLEMMESEN, R FROESE \& B UEBERSCHÄR (1991) The use of multivariate morphometrics to determine the nutritional condition of marine fish larvae. International Council for the Exploration of the Sea. 17pp.

ROCHA CFD (1989) Diet of a tropical lizard (Liolaemus lutzae) of southeastern Brazil. Journal of Herpetology 23: 292-294.

ROCHA CFD (1998) Ontogenetic shift in the rate of plant consumption in a tropical lizard (Liolaemus lutzae). Journal of Herpetology 32: 274-279.

SLATKIN M (1984) Ecological causes of sexual dimorphism. Evolution 38: 622-630.

VAN SLUYS M (1993) Food habits of the lizard Tropidurus itambere (Tropiduridae) in Southeastern Brazil. Journal of Herpetology 27: 347-351.

VEGA LE (1999) Ecología trópica de Liolaemus multimaculatus (Sauria: Tropiduridae). Bolletino dil Museo Regionale di Scienze Naturali di Torino (Italy) 16: $27-38$ 
VIANA ML, C JOVANOVICH \& P VALDÉS (1994) Hábitos alimentarios de Liolaemus darwini (Sauria: Iguanidae), en el Valle de Tin Tin, Argentina. Revista de Biología Tropical (Costa Rica) 42: 379-381.

Editor Asociado: P. Marquet

Recibido el 27 de junio de 2000; aceptado el 5 de febrero de 2001
VIDELA F (1983) Hábitos alimentarios de iguánidos del oeste árido de la Argentina. Deserta (Argentina ) 7 : 192-202.

ANEXO 1

Material examinado

Población de estepa: DB 80, 82-84, 86-87, 106, 109, 112-114,117, 122, 144-149, 152, $155,156$.

Población de bosque: DB 91, 92, 93, 94, 104, 105, 107, 108,110,118-121, 124, 126-128, 136-143. 